\title{
Interventions to improve retention-in- care and treatment adherence among patients with drug-resistant tuberculosis: a systematic review
}

\author{
Stephanie Law ${ }^{1}$, Amrita Daftary ${ }^{1,2,3}$, Max O’Donnell ${ }^{3,4}$, Nesri Padayatchi $^{3}$, \\ Liviana Calzavara ${ }^{5}$ and Dick Menzies ${ }^{1,2}$
}

Affiliations: ${ }^{1}$ Department of Epidemiology, Biostatistics and Occupational Health, McGill University, Montreal, QC, Canada. ${ }^{2}$ McGill International TB Centre, McGill University, Montreal, QC, Canada. ${ }^{3}$ CAPRISA-MRC TB-HIV Pathogenesis and Treatment Unit, Durban, South Africa. ${ }^{4}$ Division of Pulmonary, Allergy and Critical Care Medicine, and Department of Epidemiology, Mailman School of Public Health, Columbia University Medical Center, New York, NY, USA. ${ }^{5}$ Dalla Lana School of Public Health, University of Toronto, Toronto, ON, Canada.

Correspondence: Stephanie Law, 1020 Ave des Pins Ouest, Montreal, Quebec, H3A 1A2, Canada. E-mail: lawstephagmail.com

@ERSpublications

To effectively improve retention rates in the treatment of drug-resistant tuberculosis, psychosocial support, provided through one-on-one counselling and home visits, should be provided throughout treatment, rather than only during the intensive phase http://ow.ly/Yiyf30lXxPW

Cite this article as: Law S, Daftary A, O’Donnell M, et al. Interventions to improve retention-in-care and treatment adherence among patients with drug-resistant tuberculosis: a systematic review. Eur Respir J 2019; 53: 1801030 [https://doi.org/10.1183/13993003.01030-2018].

ABSTRACT The global loss to follow-up (LTFU) rate among drug-resistant tuberculosis (DR-TB) patients remains high at $15 \%$. We conducted a systematic review to explore interventions to reduce LTFU during DR-TB treatment.

We searched for studies published between January 2000 and December 2017 that provided any form of psychosocial or material support for patients with DR-TB. We estimated point estimates and 95\% confidence intervals of the proportion LTFU. We performed subgroup analyses and pooled estimates using an exact binomial likelihood approach.

We included 35 DR-TB cohorts from 25 studies, with a pooled proportion LTFU of 17 (12-23)\%. Cohorts that received any form of psychosocial or material support had lower LTFU rates than those that received standard care. Psychosocial support throughout treatment, via counselling sessions or home visits, was associated with lower LTFU rates compared to when support was provided through a limited number of visits or not at all.

Our review suggests that psychosocial support should be provided throughout DR-TB treatment in order to reduce treatment LTFU. Future studies should explore the potential of providing self-administered therapy complemented with psychosocial support during the continuation phase. 


\section{Introduction}

Approximately $15 \%$ of 1.67 million annual global deaths due to tuberculosis (TB) are from rifampicin-resistant $(\mathrm{RR})$ or multidrug-resistant (MDR)-TB, a strain resistant to at least rifampicin and isoniazid, the two most effective first-line anti-TB drugs [1]. Treating RR-/MDR-TB with second-line drugs is significantly costlier, more toxic, less effective and takes longer than treating drug-susceptible TB. Thus, rates of treatment noncompletion and interruption rates in RR-/MDR-TB are significantly higher. Approximately $15 \%$ of all RR-/MDR-TB patients are lost to follow-up from treatment, defined as interrupting treatment for $>2$ months, and only half are treated successfully [1].

Treatment nonadherence and interruption diminish the quality of life of people living with RR-/MDR-TB [2] and increase disease transmission. Developing effective interventions to improve adherence and retention in RR-/MDR-TB care is crucial. A systematic review [3] found that MDR-TB treatment strategies that used a more comprehensive approach, including financial and nutritional support, tended to have fewer losses to follow-up. However, the review, which included 75 studies, did not identify any nonobservational experimental trials for inclusion, and thus was subject to a high risk of confounding bias.

In light of increased global efforts to improve treatment and management for RR-/MDR-TB, we have synthesised new evidence, including observational and quasi-experimental studies published since the earlier systematic review, on the effectiveness of interventions in RR-/MDR-TB treatment that include various combinations of psychosocial, educational or material support. We describe and assess these interventions, their effectiveness in reducing losses to follow-up and improving adherence, and issues affecting their implementation.

\section{Methods}

This review is reported according to the PRISMA (preferred reporting items for systematic reviews and meta-analyses) statement [4], registered on the PROSPERO database (\#CRD42016052854) and analysed according to MOOSE (meta-analyses of observational studies in epidemiology) guidelines [5].

\section{Search strategy}

We searched MEDLINE (PubMed), Embase and Embase Classic, Institute for Scientific Information Web of Science, Scopus, PsycINFO, Global Health, Social Work abstracts and the Cochrane Central Register of Controlled Trials, for studies published between 2000 (the year the World Health Organization (WHO) first launched DOTS-Plus pilot projects for treatment and management of MDR-TB [6]) and December 14, 2017. Our search strategy combined the following concepts: 1) tuberculosis; 2) adherence/compliance/ default/drop-out; 3) concordance or contract; 4) linkage/referral/tracing; 5) reminder/monitor; 6) training/ education/counselling; 7) motivational/behavioural/social support; 8) patient-centred care/retention; 9) health system/services intervention/programme/strategy; 10) cash/reimbursement/refund/reward/incentives; 11) dietary/nutritional supplement or food; 12) directly observed therapy (DOT); and 13) evaluation (online supplementary table S1 presents search details). No geographical or language restrictions were applied. We identified additional articles from reference lists of identified original articles and four recent systematic reviews on strategies for reducing MDR-TB patient losses to follow-up [3]; decentralised models of MDR-TB care [7]; community-based MDR-TB treatment [8]; and DOT in MDR-TB treatment [9].

\section{Study screening and eligibility criteria}

We included primary studies that 1) reported final treatment outcomes including losses to follow-up; and 2) examined a health services intervention targeting patients with RR-/MDR-TB that included at least some form of psychosocial, educational or material support. We defined each as any support provided to address psychological or social issues; any education or counselling provided pertaining to TB treatment; and any nutritional (e.g. food package or hot meals) or financial (e.g. reimbursement for treatment-related expenses and lost wages) support. Studies only examining surgical or drugs-related interventions were not included; other exclusion criteria included 1) $<10$ cases of RR-/MDR-TB; 2 ) only children (aged $<18$ years) included, due to their likely dependence on adult caregivers for adherence; 3) only interim outcomes reported (defined as outcomes, such as 6-month sputum conversion, that occurred before the planned end of treatment); 4) no details on drug susceptibility testing for at least rifampicin; or 5) no treatment with second-line drugs. One reviewer (SL) screened all titles and abstracts, and two independent reviewers (SL and $\mathrm{AD}$ ) screened full reports of potentially relevant studies; discrepancies were resolved by discussion. We contacted authors of published abstracts and studies to obtain further information when necessary.

\section{Types of outcome measures}

Our primary outcome of interest was loss to follow-up, defined as treatment interruption for $\geqslant 2$ months [1]. Secondary outcomes included any measures of treatment adherence. 


\section{Data extraction and analysis}

One reviewer (SL) extracted outcomes data, participant characteristics, details on study interventions and information necessary to assess study quality. We used the ROBINS-I tool [10] to assess the quality of cohort studies, and the Cochrane Risk of Bias Tool [11] for quasi-experimental trials. All extracted data were entered into Excel (Microsoft Corporation, Redmond, WA, USA).

We estimated unadjusted risk ratios and $95 \%$ confidence intervals to compare the proportions lost to follow-up in each arm/cohort in comparative studies. We conducted pooled analyses of all study cohorts to analyse the association between different types of support and losses to follow-up. We used the exact binomial likelihood approach, including a random effect to account for between-study heterogeneity, to estimate pooled proportions lost to follow-up and 95\% confidence intervals. This approach produces less-biased estimates of the pooled effect and the between-study variability than normal approximation approaches [12]. We investigated heterogeneity using the $\mathrm{I}^{2}$ statistic via subgroup analyses, and explored differences in geographic regions, extensively drug-resistant (XDR) status, HIV prevalence, previous treatment, treatment delivery methods and types of adherence support. Cochran's Q test was performed to test for subgroup differences. In our main analyses, we excluded patients who died or failed treatment (to exclude from the denominator patients who could not have experienced the outcome of lost to follow-up) and patients who were transferred out or not evaluated for final treatment outcomes. We conducted sensitivity analyses in which we considered patients who were transferred out or not evaluated, or those who died, as patients lost to follow-up. All statistical analyses were performed in $\mathrm{R}$ (www.r-project.org).

\section{Results}

\section{Description of included studies}

Our search strategy identified 5911 studies; of these we included 23 cohort studies and two quasi-experimental trials [13-37] in our analyses (figure 1). These 25 studies included 35 different cohorts of RR-/MDR-TB patients, distinguished by the different types and levels of adherence support. The types of treatment support provided to the included cohorts are summarised in table 1 . All but three studies [20, $22,37]$ were conducted in high-burden TB/MDR-TB countries [1].

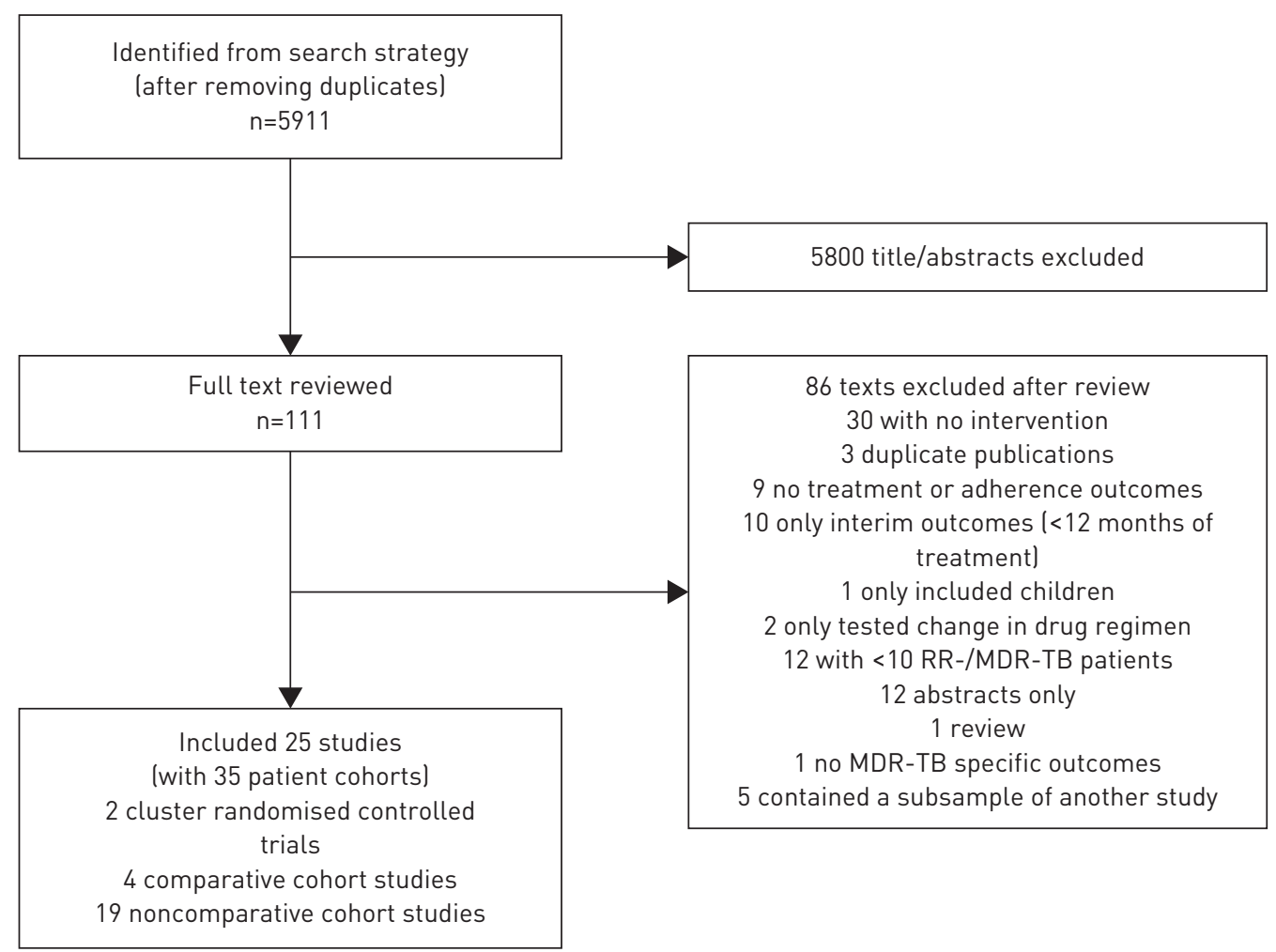

FIGURE 1 Flow diagram of literature search and study selection. RR: rifampicin-resistant; MDR: multidrug-resistant; TB: tuberculosis. 
TABLE 1 Description of treatment support provided in included study cohorts

\begin{tabular}{|c|c|c|c|c|c|c|c|c|c|c|}
\hline $\begin{array}{l}\text { FiRST AUTHOR, } \\
\text { year [ref.] }\end{array}$ & $\begin{array}{c}\text { Subjects } \\
n\end{array}$ & $\begin{array}{c}\text { Country, } \\
\text { study period }\end{array}$ & $\begin{array}{l}\text { DOT frequency } \\
\text { (intensive/ } \\
\text { continuation } \\
\text { phase) }\end{array}$ & DOT location & Individual counselling & Home visits available & $\begin{array}{l}\text { Financial support } \\
\text { offered }\end{array}$ & $\begin{array}{l}\text { Food packages } \\
\text { provided }\end{array}$ & $\begin{array}{l}\text { Group } \\
\text { counselling }\end{array}$ & $\begin{array}{l}\text { Counselling/ } \\
\text { education } \\
\text { offered to family }\end{array}$ \\
\hline \multicolumn{11}{|c|}{ Studies with two or more patient cohorts } \\
\hline $\begin{array}{l}\text { BARAL, } 2014 \text { [13] } \\
\text { (control)" }\end{array}$ & 33 & Nepal, 2008 & Daily/daily & Clinic & No & No & No & No & No & NS \\
\hline $\begin{array}{l}\text { BARAL, } 2014[13] \\
\text { (arm 1) }\end{array}$ & 33 & Nepal, 2008 & Daily/daily & Clinic & $\begin{array}{c}2-5 \text { tailored sessions by trained } \\
\text { nurse }\end{array}$ & No & No & No & Every 2-3 weeks & NS \\
\hline $\begin{array}{l}\text { BARAL, } 2014 \text { [13] } \\
\text { (arm 2) }\end{array}$ & 42 & Nepal, 2008 & Daily/daily & Clinic & $\begin{array}{c}2-5 \text { tailored sessions by trained } \\
\text { nurse }\end{array}$ & No & NPR 2000 per month & No & Every 2-3 weeks & NS \\
\hline $\begin{array}{l}\text { Cox, } 2014[14] \\
\text { (control) }\end{array}$ & 216 & $\begin{array}{l}\text { South Africa, } \\
2005-2010\end{array}$ & Daily/daily & $\begin{array}{l}\text { In-patient, } \\
\text { hospital/clinic }\end{array}$ & NS & No & No & No & No & No \\
\hline $\begin{array}{l}\text { Cox, } 2014[14] \\
\text { (intervention)" }\end{array}$ & 571 & $\begin{array}{l}\text { South Africa, } \\
2005-2010\end{array}$ & Daily/daily & Clinic & $\begin{array}{c}\text { Routine counselling at start of } \\
\text { treatment }\end{array}$ & $\begin{array}{l}\text { No, but home } \\
\text { assessment performed by } \\
\text { CHW at start of treatment }\end{array}$ & No & No & $\begin{array}{l}\text { Weekly peer } \\
\text { support groups }\end{array}$ & NS \\
\hline $\begin{array}{l}\text { Huerga, } 2017 \text { [15] } \\
\text { (Homa Bay) }\end{array}$ & 28 & $\begin{array}{l}\text { Kenya, 2006- } \\
2012\end{array}$ & $\begin{array}{l}\text { Twice daily/twice } \\
\text { daily }\end{array}$ & Clinic/home & $\begin{array}{l}\text { Weekly to monthly counselling } \\
\text { sessions, and as needed }\end{array}$ & Daily home visits by CHW & Rent and travel & No & No & NS \\
\hline $\begin{array}{l}\text { HuERGA, } 2017 \text { [15] } \\
\text { (Mathare) }\end{array}$ & 70 & $\begin{array}{l}\text { Kenya, 2006- } \\
2012\end{array}$ & Daily/daily & Clinic & $\begin{array}{l}\text { Weekly to monthly counselling } \\
\text { sessions, and as needed }\end{array}$ & No & $\begin{array}{l}\text { Rent and travel } \\
\text { expenses }\end{array}$ & $\begin{array}{l}\text { Daily hot meal and } \\
\text { monthly food basket }\end{array}$ & No & NS \\
\hline $\begin{array}{l}\text { HuERGA, } 2017[15] \\
\text { (Nairobi) }\end{array}$ & 71 & $\begin{array}{l}\text { Kenya, 2006- } \\
2012\end{array}$ & Daily/daily & Clinic & $\begin{array}{l}\text { Counselling by nurses on request } \\
\text { by doctors }\end{array}$ & No & $\begin{array}{l}\text { Rent and travel } \\
\text { expenses }\end{array}$ & No & No & NS \\
\hline $\begin{array}{l}\text { LovedAY, } 2015[16] \\
\text { (hospital)" }\end{array}$ & 813 & $\begin{array}{l}\text { South Africa, } \\
2008-2010\end{array}$ & Daily/none & $\begin{array}{l}\text { In-patient, } \\
\text { hospital }\end{array}$ & NS & No & No & No & No & NS \\
\hline $\begin{array}{l}\text { LovEDAY, } 2015[16] \\
\text { (site 1) }\end{array}$ & 125 & $\begin{array}{l}\text { South Africa, } \\
2008-2010\end{array}$ & Daily/daily & Clinic/home & Weekly educational sessions & Daily home visits by $\mathrm{CHW}$ & Travel expenses & No & No & Yes \\
\hline $\begin{array}{l}\text { LovEDAY, } 2015[16] \\
\text { (sites } 2 \text { and } 3 \text { ) }\end{array}$ & 350 & $\begin{array}{l}\text { South Africa, } \\
2008-2010\end{array}$ & Daily/none & Clinic/home & $\begin{array}{l}\text { Unspecified frequency and } \\
\text { duration }\end{array}$ & No & Travel expenses & No & No & Yes \\
\hline $\begin{array}{l}\text { LovEDAY, } 2015[16] \\
\text { (site 4) }\end{array}$ & 261 & $\begin{array}{l}\text { South Africa, } \\
2008-2010\end{array}$ & Daily/none & Clinic & $\begin{array}{l}\text { Unspecified frequency and } \\
\text { duration }\end{array}$ & No & Travel expenses & No & No & Yes \\
\hline $\begin{array}{l}\text { MoнR, } 2017 \text { [17] } \\
\text { (SAT) }\end{array}$ & 244 & $\begin{array}{l}\text { South Africa, } \\
2010-2014\end{array}$ & Daily/none & Clinic & $\begin{array}{l}4 \text { standardised sessions during } \\
\text { intensive phase, and } 1 \text { at start of } \\
\text { continuation phase }\end{array}$ & $\begin{array}{l}\text { Weekly visits by } \mathrm{CHW} \text { at } \\
\text { the start of continuation } \\
\text { phase, monthly after }\end{array}$ & No & No & No & NS \\
\hline $\begin{array}{l}\text { MoнR, } 2017 \text { [17] } \\
\text { (SOC) }\end{array}$ & 160 & $\begin{array}{l}\text { South Africa, } \\
2010-2014\end{array}$ & Daily/daily & Clinic & $\begin{array}{l}4 \text { standardised sessions during } \\
\text { intensive phase }\end{array}$ & No & No & No & No & NS \\
\hline $\begin{array}{l}\text { TANEJA, } 2017[18] \\
\text { (control) }\end{array}$ & 50 & India, 2014 & $\begin{array}{l}\text { Thrice weekly/ } \\
\text { none }\end{array}$ & $\begin{array}{l}\text { Health facility } \\
\text { (public/private/ } \\
\text { NGO) }\end{array}$ & $\begin{array}{l}\text { Thrice weekly during intensive } \\
\text { phase, weekly thereafter }\end{array}$ & No & No & No & No & NS \\
\hline $\begin{array}{l}\text { TANEJA, } 2017 \text { [18] } \\
\text { (intervention) }\end{array}$ & 50 & India, 2014 & $\begin{array}{l}\text { Thrice weekly/ } \\
\text { none }\end{array}$ & $\begin{array}{l}\text { Health facility } \\
\text { (public/private/ } \\
\text { NGO) }\end{array}$ & $\begin{array}{l}\text { Fortnightly at home and thrice } \\
\text { weekly at clinic during intensive } \\
\text { phase, weekly at clinic and every } \\
45 \text { days at home thereafter }\end{array}$ & $\begin{array}{l}\text { Fortnightly visits from } \\
\text { homecare team during } \\
\text { intensive phase, and } \\
\text { every } 45 \text { days thereafter }\end{array}$ & No & $\begin{array}{l}\text { Daily provision of eggs } \\
\text { and multigrain biscuits }\end{array}$ & No & Yes \\
\hline \multicolumn{11}{|l|}{$\begin{array}{l}\text { Studies with a single } \\
\text { patient cohort }\end{array}$} \\
\hline ALenE, 2017 [19] & 481 & $\begin{array}{l}\text { China, 2011- } \\
2014\end{array}$ & Daily/daily & Clinic/home & $\begin{array}{l}\text { Throughout initial hospitalisation } \\
\text { (1-2 months) }\end{array}$ & None specified & No & No & No & Yes \\
\hline $\begin{array}{l}\text { BASTARD, } 2015 \\
\text { [20] }\end{array}$ & 403 & $\begin{array}{l}\text { Armenia/ } \\
\text { Georgia, 2002- } \\
2010\end{array}$ & Daily/daily & Clinic/home & Routine sessions & $\begin{array}{l}\text { Daily by health personnel } \\
\text { or CHW }\end{array}$ & Travel expenses & Yes, unspecified & Yes, unspecified & NS \\
\hline \multirow[t]{2}{*}{ Cox, 2007 [21] } & 87 & $\begin{array}{l}\text { Uzbekistan, } \\
\text { 2003-2005 }\end{array}$ & Daily/daily & Clinic & Daily counselling, or as needed & No & Travel expenses & $\begin{array}{l}4 \text { meals daily during } \\
\text { hospitalisation; } \\
\text { monthly food parcels } \\
\text { after }\end{array}$ & No & NS \\
\hline & 25 & & Daily/none & & & No & No & No & No & NS \\
\hline
\end{tabular}




\begin{tabular}{|c|c|c|c|c|c|c|c|c|c|c|}
\hline $\begin{array}{l}\text { FiRST AUTHOR, } \\
\text { year [ref.] }\end{array}$ & $\begin{array}{l}\text { Subjects } \\
n\end{array}$ & $\begin{array}{l}\text { Country, } \\
\text { study period }\end{array}$ & $\begin{array}{l}\text { DOT frequency } \\
\text { (intensive/ } \\
\text { continuation } \\
\text { phase) }\end{array}$ & DOT location & Individual counselling & Home visits available & $\begin{array}{l}\text { Financial support } \\
\text { offered }\end{array}$ & $\begin{array}{l}\text { Food packages } \\
\text { provided }\end{array}$ & $\begin{array}{c}\text { Group } \\
\text { counselling }\end{array}$ & $\begin{array}{l}\text { Counselling/ } \\
\text { education } \\
\text { offered to family }\end{array}$ \\
\hline $\begin{array}{l}\text { EscUDERo, } 2006 \\
\text { [22] }\end{array}$ & & $\begin{array}{l}\text { Spain, 1998- } \\
\quad 2000\end{array}$ & & $\begin{array}{l}\text { In-patient, } \\
\text { hospital }\end{array}$ & $\begin{array}{c}\text { Repeatedly during hospitalisation, } \\
\text { monthly thereafter, by clinician/ } \\
\text { psychologist }\end{array}$ & & & & & \\
\hline $\begin{array}{l}\text { Gelmanova, } 2011 \\
\text { [23] }\end{array}$ & 38 & $\begin{array}{l}\text { Russia, 2006- } \\
2008\end{array}$ & $\begin{array}{l}\text { Twice daily/twice } \\
\text { daily }\end{array}$ & Hospital/Home & $\begin{array}{l}\text { Daily counselling, or as needed, } \\
\text { by nurses and psychologist }\end{array}$ & $\begin{array}{l}\text { Twice daily by a team of } \\
\text { two nurses }\end{array}$ & Travel passes & Daily food parcels & No & Yes \\
\hline $\begin{array}{l}\text { ISAAKIDIS, } 2011 \\
\text { [24] }\end{array}$ & 35 & $\begin{array}{l}\text { India, 2007- } \\
\quad 2011\end{array}$ & $\begin{array}{l}\text { Twice daily/twice } \\
\text { daily }\end{array}$ & $\begin{array}{l}\text { Health facility } \\
\text { (public/private/ } \\
\text { NGO) }\end{array}$ & Monthly psychosocial follow-up & No & No & No & No & NS \\
\hline JosEPH, 2011 [25] & 38 & $\begin{array}{l}\text { India, 2006- } \\
\quad 2007\end{array}$ & Daily/daily & $\begin{array}{c}\text { Health facility } \\
\text { (public/private/ } \\
\text { NGO) }\end{array}$ & $\begin{array}{l}\text { Initial education by medical } \\
\text { officer and social worker, } \\
\text { followed by daily adherence } \\
\text { advice from trained DOT provider }\end{array}$ & No & No & No & No & Yes \\
\hline $\begin{array}{l}\text { KeshavjeE, } 2008 \\
\quad[26]\end{array}$ & 608 & $\begin{array}{l}\text { Russia, 2000- } \\
\quad 2004\end{array}$ & Daily/daily & $\begin{array}{l}\text { In-patient, } \\
\text { hospital; clinic/ } \\
\text { rural health } \\
\text { outpost }\end{array}$ & Daily counselling, or as needed & No & No & $\begin{array}{l}\text { Monthly food packages } \\
\text { and meals for adherent } \\
\text { patients }\end{array}$ & No & NS \\
\hline KLIIMAN, 2009 [27] & 235 & $\begin{array}{l}\text { Estonia, 2003- } \\
2005\end{array}$ & Daily/daily & $\begin{array}{c}\text { In-patient, } \\
\text { hospital; clinic }\end{array}$ & NS & No & Travel expenses & Yes, unspecified & No & NS \\
\hline $\begin{array}{l}\text { MeressA, } 2015 \\
{[28]}\end{array}$ & 612 & $\begin{array}{l}\text { Ethiopia, 2009- } \\
\quad 2014\end{array}$ & Daily/daily & Clinic/home & Monthly counselling & $\begin{array}{l}\text { Monthly visits by } \\
\text { outpatient team }\end{array}$ & $\begin{array}{l}\text { Rent and travel } \\
\text { expenses }\end{array}$ & Monthly food baskets & No & Yes \\
\hline MitNick, 2003 [29] & 75 & $\begin{array}{l}\text { Peru, 1996- } \\
\quad 1999\end{array}$ & Daily/daily & Clinic/home & Daily counselling, or as needed & Daily by $\mathrm{CHW}$ & Travel expenses & Yes, unspecified & $\begin{array}{l}\text { Weekly, bimonthly } \\
\text { social support } \\
\text { groups }\end{array}$ & Yes \\
\hline Мitnick, 2008 [30] & 650 & $\begin{array}{l}\text { Peru, 1999- } \\
\quad 2002\end{array}$ & Daily/daily & Clinic/home & Daily counselling, or as needed & Daily by $\mathrm{CHW}$ & Travel expenses & Yes, unspecified & $\begin{array}{l}\text { Weekly, bimonthly } \\
\text { social support } \\
\text { groups }\end{array}$ & Yes \\
\hline МонR, 2015 [31] & 853 & $\begin{array}{l}\text { South Africa, } \\
2008-2012\end{array}$ & Daily/daily & Clinic & $\begin{array}{l}3 \text { sessions in intensive phase and } \\
1 \text { in continuation phase }\end{array}$ & No & No & Yes, unspecified & $\begin{array}{l}\text { Weekly } \\
\text { peer-support } \\
\text { groups }\end{array}$ & Yes \\
\hline SATTI, 2012 [32] & 134 & $\begin{array}{l}\text { Lesotho, 2008- } \\
2009\end{array}$ & $\begin{array}{l}\text { Twice daily/twice } \\
\text { daily }\end{array}$ & Home & Daily counselling, or as needed & $\begin{array}{l}\text { Twice daily by trained } \\
\text { CHW }\end{array}$ & Travel expenses & Monthly food packages & No & Yes \\
\hline SHIN, 2006 [33] & 244 & $\begin{array}{l}\text { Russia, } 1998- \\
2000\end{array}$ & Daily/daily & $\begin{array}{c}\text { Clinic/rural } \\
\text { health outpost }\end{array}$ & Daily counselling, or as needed & No & No & $\begin{array}{l}\text { Monthly food packages/ } \\
\text { meals for adherent } \\
\text { patients }\end{array}$ & No & NS \\
\hline SuÁrez, 2002 [34] & 298 & $\begin{array}{l}\text { Peru, 1997- } \\
\quad 1999\end{array}$ & Daily/daily & Clinic & NS & No & No & Weekly food parcels & NS & No \\
\hline Тномаs, 2007 [35] & 66 & $\begin{array}{l}\text { India, 1999- } \\
2003\end{array}$ & $\begin{array}{l}\text { Thrice weekly/ } \\
\text { thrice weekly }\end{array}$ & $\begin{array}{l}\text { Health facility } \\
\text { (public/private/ } \\
\text { NGO) }\end{array}$ & Monthly sociological counselling & No & $\begin{array}{l}\text { Monthly } \\
\text { compensation for lost } \\
\text { wages and travel } \\
\text { expenses }\end{array}$ & No & No & NS \\
\hline VAGHELA, 2015 [36] & 101 & $\begin{array}{l}\text { India, 2009- } \\
\quad 2010\end{array}$ & Daily/daily & $\begin{array}{l}\text { Health facility } \\
\text { (public/private/ } \\
\text { NGO) }\end{array}$ & $\begin{array}{l}\text { Every } 15 \text { days during intensive } \\
\text { phase, every } 45 \text { days thereafter }\end{array}$ & $\begin{array}{l}\text { Visits by } \mathrm{CHW} \text { every } \\
15 \text { days during intensive } \\
\text { phase, every } 45 \text { days } \\
\text { thereafter }\end{array}$ & None & $\begin{array}{l}\text { Daily provision of eggs } \\
\text { and multigrain biscuits }\end{array}$ & No & Yes \\
\hline Yu, 2015 [37] & 126 & $\begin{array}{l}\text { Taiwan, 2007- } \\
2009\end{array}$ & $\begin{array}{l}\text { Twice daily/twice } \\
\text { daily }\end{array}$ & Clinic/home & Daily counselling, or as needed & $\begin{array}{l}\text { Daily visits by medical } \\
\text { team }\end{array}$ & Monthly income & No & No & Yes \\
\hline
\end{tabular}




\section{Quality of studies}

We did not exclude any study based on our assessment of quality (see online supplementary tables S4 and S5 for summaries and online supplementary tables S9 and S10 for details). All included studies used routinely collected data within local TB systems to ascertain treatment outcomes. Reporting of intervention details varied across studies, and it was difficult to evaluate the fidelity of intervention implementation and delivery in three studies $[19,20,25]$. Some studies did not provide important patient characteristics, such as any previous TB treatment $(n=2)$, previous treatment with second-line drugs $(n=13)$, XDR status $(n=6)$ and HIV status $(n=6)$; these studies were conducted in settings where $<10 \%$ of TB patients are infected with HIV) (online supplementary table S2).

Five cohort studies included two or more separate cohort groups [14-17, 37], which allowed for comparison of outcomes. However, we excluded the control cohort in the study by Yu et al. [37] because we were not able to obtain adequate details on the care provided, nor the number of patients lost to follow-up. Of the remaining four studies, two compared patient cohorts before and after implementation of an intervention $[14,17]$ and two analysed concurrent cohort groups receiving different types of care $[15,16]$. None of the studies provided adjusted estimates for the effect of intervention on loss to follow-up rates to account for potential confounding. All four studies were considered to have serious risks of biases due to confounding and two were considered to have moderate risks of bias due to missing data (patients who were transferred out or not evaluated for final treatment outcomes) (online supplementary table S4).

Two trials were included in our analysis $[13,18]$. Both were cluster randomised trials where healthcare facilities were randomised to provide routine care or the study interventions. The overall risk of performance bias was high for both studies, because sites selected to implement the intervention were unblinded (online supplementary table S5). This could have a spillover effect and positively affect standard elements of care, thereby possibly overestimating the benefit of the intervention. However, sites providing routine care could have improved their care to compensate for the absence of an intervention, thereby underestimating the benefit. Furthermore, due to the small number of clusters randomised in each study, patient and site characteristics were not balanced between the intervention and control arms, which could lead to residual confounding. BARAL et al. [13] adjusted for age and sex, but neither study accounted for clustering by site or other important baseline confounders.

\section{Results of head-to-head comparisons}

The results from comparative cohort studies and trials are shown in figure 2. The standard of care (study control) varied across the studies (see table 1 and online supplementary table S8 for details). Given the variation in the control groups as well as the types of support provided in the intervention groups, pooling of intervention effects was not possible. Patients who received some form of psychosocial, educational or material support, in addition to the standard care, were less likely to be lost to follow-up, with the exception of the study by Cox et al. [14]. In their pilot intervention study, Cox et al. [14] found no difference (risk ratio 1.04, 95\% CI 0.83-1.32) between the control group, which received hospital-initiated MDR-TB treatment, and the intervention group, which received community-based, clinic-initiated

Control Intervention LTFU/total LTFU/total

$\begin{array}{lccc}\text { BarAL, 2014 (RCT) [13] } & \text { Clinic DOT versus clinic DOT+counselling } & 15 / 69 & 2 / 30 \\ \text { BarAL, 2014 (RCT) [13] } & \text { Clinic DOT versus clinic DOT+counselling+monthly income } & 15 / 69 & 6 / 38 \\ \text { Cox, 2014 (cohort) [14] } & \text { Hospital versus clinic DOT+counselling+support group } & 152 / 387 & 59 / 144 \\ \text { Huerga, } 2017 \text { (cohort) [15] } & \text { Clinic DOT versus clinic DOT+counselling+food baskets } & 6 / 49 & 6 / 58 \\ \text { Huerga, 2017 (cohort) [15] } & \text { Clinic DOT versus clinic/home DOT+counselling+home visits } & 6 / 49 & 0 / 16 \\ \text { LovedAy, 2015 (cohort) [16] } & \text { Hospital versus clinic DOT } & 230 / 669 & 28 / 163 \\ \text { LovedAy, 2015 (cohort) [16] } & \text { Hospital versus clinic/home DOT } & 230 / 669 & 70 / 272 \\ \text { LovedAy, 2015 (cohort) [16] } & \text { Hospital versus clinic/home DOT+counselling+home visits } & 230 / 669 & 9 / 99 \\ \text { Mohr, 2017 (cohort) [17] } & \text { Clinic DOT+counselling versus SAT+counselling+home visits } & 44 / 110 & 47 / 146 \\ \text { TaneJA, 2017 (RCT) [18] } & \text { Facility DOT versus facility DOT+home visits+food baskets } & 21 / 35 & 22 / 42\end{array}$

BARAL, 2014 (RCT) [13]

BARAL, 2014 (RCT) [13]

Cox, 2014 (cohort) [14]

LovedAy, 2015 (cohort) [16]

LOVEDAY, 2015 (cohort) [16]

MoHR, 2017 (cohort) [17]

TANEJA, 2017 (RCT) [18]
$21 / 35$
$22 / 42$

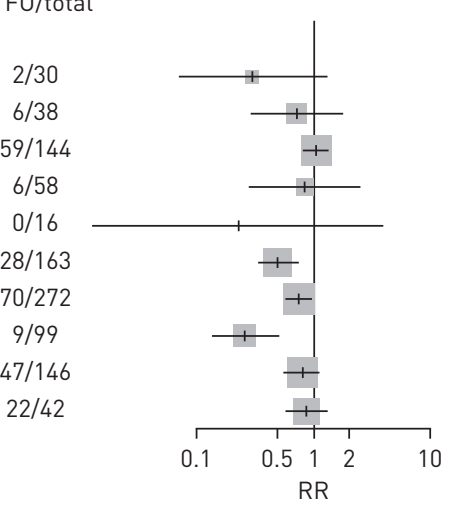

$\operatorname{RR}(95 \% \mathrm{CI})$

$0.31(0.07-1.26)$

$0.73(0.31-1.72)$

$1.04(0.83-1.32)$

$0.84(0.29-2.45)$

0.23 (0.01-3.88)

$0.50(0.35-0.71)$

0.75 (0.60-0.94)

$0.26(0.14-0.50)$

$0.80(0.58-1.12)$

$0.87(0.59-1.30)$

FIGURE 2 Forest plot of unadjusted risk ratios (RR) comparing proportions lost to follow-up (LTFU) between control and intervention arms in comparative studies (two or more patient cohorts). The size of the square is proportional to the size of the study sample. Patients who died, failed treatment, who transferred out or whose treatment outcome was not evaluated were excluded from the denominator. See table 1 and online supplementary table S8 for details of treatment delivery and management for each study. Data are presented as $\mathrm{n}$ unless otherwise stated. DOT: directly observed therapy; SAT: self-administered therapy. 
treatment with routine counselling sessions and access to a peer-support group. The greatest reduction in losses to follow-up was seen in two cohorts that received support through daily home visits by community health workers, as well as home-based DOT, when compared to the standard of care $[15,16]$. In the cluster randomised trial by BARAL et al. [13], the addition of individually tailored counselling sessions provided by nurses reduced the risk of lost to follow-up by $70 \%$, but this estimate was very imprecise (risk ratio $0.31,95 \%$ CI $0.07-1.26$ ), and adding a monthly income supplement did not improve the effect (risk ratio 0.73 , 95\% CI $0.31-1.72$ ).

\section{Pooled results across studies}

Results from all study cohorts, in both comparative and noncomparative studies, that received any form of psychosocial, educational or material support were pooled in the following analysis (online supplementary table S2 presents the characteristics of included study cohorts). We excluded the standard of care or control groups in two studies that did not provide any psychosocial, educational or material support [13, 16], as well as one comparative cohort study [14] because its intervention group was a subsample of a larger single-cohort study [31].

Final treatment outcomes were reported for a total of 6655 RR-/MDR-TB patients in 31 included study cohorts (online supplementary table S3). After excluding patients who died, failed treatment or were transferred out or not evaluated for final treatment outcomes, there remained a total of 5114 patients (median of 84 patients per study cohort). The pooled proportion lost to follow-up was 17\% (95\% CI 12 $23 \%$ ), as seen in the forest plot (figure 3). Study heterogeneity was high across all included study cohorts $\left(\mathrm{I}^{2}=96 \%\right)$, and remained high in subgroup analyses by WHO region (except in the Americas region, probably because all three cohorts were largely based in Lima, Peru, within approximately the same period), HIV infection rate, proportion of patients with XDR-TB, previous TB treatment (with or without second-line drugs) and start year of the study (figure 4).

In subgroup analyses, study cohorts with more frequent contact with health workers throughout treatment (in the form of DOT visits, home visits or individual counselling sessions) tended to have fewer losses to follow-up (figure 5). Additionally, provision of financial support to reimburse rent or travel expenses, as well as to compensate lost wages during treatment, was associated with fewer losses to follow-up. There was weak evidence of any association between providing food packages, group counselling or counselling to family members and losses to follow-up. In order to distinguish the effect of frequent DOT from that of adherence support, subgroup analyses according to types of support provided were restricted to study cohorts that received either twice-daily or daily DOT throughout treatment (figure 6). Within these cohorts, those that received individual counselling throughout treatment $[15,16,21,23-26,28-30,32,33$, $36,37]$ had fewer losses to follow-up than those that received a fixed $[13,17,19,20,31]$ or unspecified $[15,27,34]$ number of individual counselling sessions at the start of treatment. Similarly, those that received any home visits by health workers had fewer losses to follow-up [15, 16, 20, 23, 29, 30, 32, 37]. Sensitivity analyses where patients who were transferred out (online supplementary figures S20-S22), or who died (online supplementary figures S23-S25) were considered as lost to follow-up yielded similar results. Furthermore, the findings remained consistent across strata of study cohorts stratified by prevalence of HIV co-infection (online supplementary figures S26-S29) and previous TB treatment (online supplementary figures S30-S33).

\section{Other adherence outcomes}

Three studies additionally reported the proportion of doses taken (or missed) by patients (online supplementary table S6), two of which did not include a comparison control group. In comparing treatment adherence before and after patients were enrolled into the study intervention, GeLMANOva et al. [23] found an increase in proportion of doses taken from 52.2\% (95\% CI $47.5-56.9 \%$ ) to $81.4 \%$ (95\% CI $76.8-$ 86.0\%). These patients received increased staff time from nurses, as well as expanded access to psychosocial support, after study enrolment.

\section{Feasibility of implementation of interventions}

A summary of feasibility and implementation issues associated with study interventions is provided in online supplementary table S7. Reported issues included reluctance from health providers to follow new intervention-directed procedures [16, 17]; difficulties identifying and training support workers [29, 35]; and lack of clarity in intervention implementation [16]. Among studies that reported on cost-effectiveness, all found that the study intervention reduced losses to follow-up and was more cost-effective than the standard treatment practices in their respective setting [23, 29, 34]. 
First author, year (cohort) [ref.]

Studies with two or more arms/cohorts BARAL, 2014 (arm 1) [13]

BARAL, 2014 (arm 2) [13]

Huerga, 2017 (Homa Bay) [15]

Huerga, 2017 (Mathare) [15]

Huerga, 2017 (Nairobi) [15]

Loveday, 2015 (site 1) [16]

Loveday, 2015 (site 2 and 3) [16]

LovedAY, 2015 (site 4) [16]

MoHR, 2017 (SAT) [17]

MoнR, 2017 (SOC) [17]

TANEJA, 2017 (control) [18]

TANEJA, 2017 (intervention) [18]

Random effects model

Heterogeneity $\mathrm{I}^{2}=93 \%, \tau^{2}=0.9430, p<0.01$

Studies with a single cohort

Alene, 2017 [19]

BASTARD, 2015 [20]

Cox, 2007 [21]

ESCUDERO, 2006 [22]

GeLmANOVA, 2011 [23]

ISAAKIDIS, 2011 [24]

JoSEPH, 2011 [25]

KeshaVJeE, 2008 [26]

KLIIMAN, 2009 [27]

Meressa, 2015 [28]

Mitnick, 2003 [29]

MITNICK, 2008 [30]

MoHr, 2015 [31]

SATTI, 2012 [32]

SHIN, 2006 [33]

SUAREZ, 2002 [34]

THOMAS, 2007 [35]

VAGHELA, 2015 [36]

Yu, 2015 [37]

Random effects mode

Heterogeneity $\mathrm{I}^{2}=97 \%, \tau^{2}=0.0355, p<0.01$

Random effects mode

Heterogeneity $\left.\right|^{2}=96 \%, \tau^{2}=1.0401, p<0.01$
Subjects n LTFU n

Proportion (95\% CI)

$\begin{array}{rr}30 & 2 \\ 38 & 6 \\ 16 & 0 \\ 58 & 6 \\ 49 & 6 \\ 99 & 9 \\ 272 & 70 \\ 163 & 28 \\ 146 & 47 \\ 110 & 44 \\ 35 & 21 \\ 42 & 22 \\ 1058 & 261\end{array}$

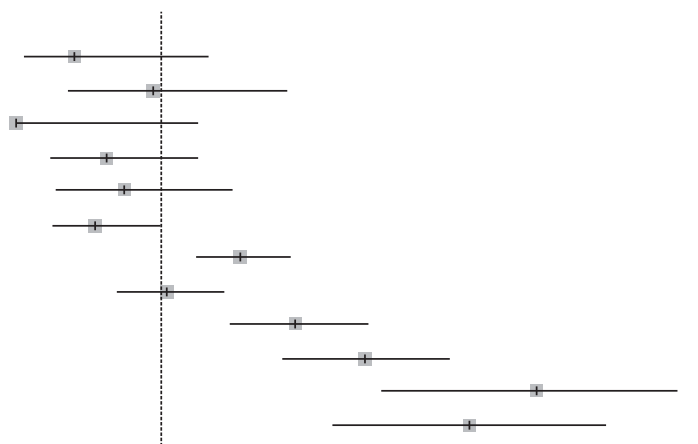

$0.07(0.01-0.22)$

$0.16(0.06-0.31)$

$0.00(0.00-0.21)$

$0.10(0.04-0.21)$

$0.12(0.05-0.25)$

$0.09(0.04-0.17)$

$0.26(0.21-0.31)$

$0.17(0.12-0.24)$

$0.32(0.25-0.40)$

$0.40(0.31-0.50)$

$0.60(0.42-0.76)$

$0.52(0.36-0.68)$

$0.20(0.12-0.32)$

$0.32(0.28-0.37)$

$0.43(0.37-0.48)$

$0.18(0.10-0.30)$

$0.09(0.01-0.28)$

$0.18(0.07-0.35)$

$0.35(0.15-0.59)$

$0.17(0.06-0.35)$

$0.23(0.19-0.27)$

$0.23(0.18-0.30)$

$0.07(0.05-0.10)$

$0.08(0.03-0.18)$

$0.13(0.10-0.16)$

$0.39(0.35-0.43)$

$0.01(0.00-0.06)$

$0.13(0.09-0.18)$

$0.12(0.09-0.17)$

$0.39(0.24-0.55)$

$0.09(0.04-0.17)$

$0.00(0.00-0.03)$

$0.15(0.10-0.22)$

$0.17(0.12-0.23)$

FIGURE 3 Forest plot of proportions lost to follow-up (LTFU) across all study cohorts. Patients who died, failed treatment, who transferred out or whose treatment outcome was not evaluated were excluded. In studies with more than one cohort, each cohort is shown separately. SAT: selfadministered therapy; SOC: standard of care.

\section{Discussion}

Strategies to improve retention-in-care and treatment adherence among disease-resistant TB patients are greatly needed to increase treatment success rates globally. This review found a broad range of adherence support interventions, all of which included some degree of educational and psychosocial counselling, as well as a variety of material support. However, very few studies reported on adherence outcomes in addition to patient losses to follow-up.

Our review found individual counselling support and home visits by health workers, provided throughout treatment, were associated with fewer losses to follow-up than when they were provided only at the start of treatment, or not at all. This association remained even after restricting the analyses to studies that provided daily DOT throughout treatment. Thus, although our study found lower rates of loss to follow-up among studies that provided more frequent DOT, this could be conflated with the associated frequency of contact with providers as well as psychosocial or educational support. This is supported by findings from МонR et al. [17], which showed that self-administered therapy, supplemented with routine home visits by community health workers during the continuation phase yielded a similar rate of loss to follow-up compared to daily clinic-based DOT without home visits. Furthermore, Gelmanova et al. [23] showed significant improvements in treatment adherence rates among MDR-TB patients when staff time 


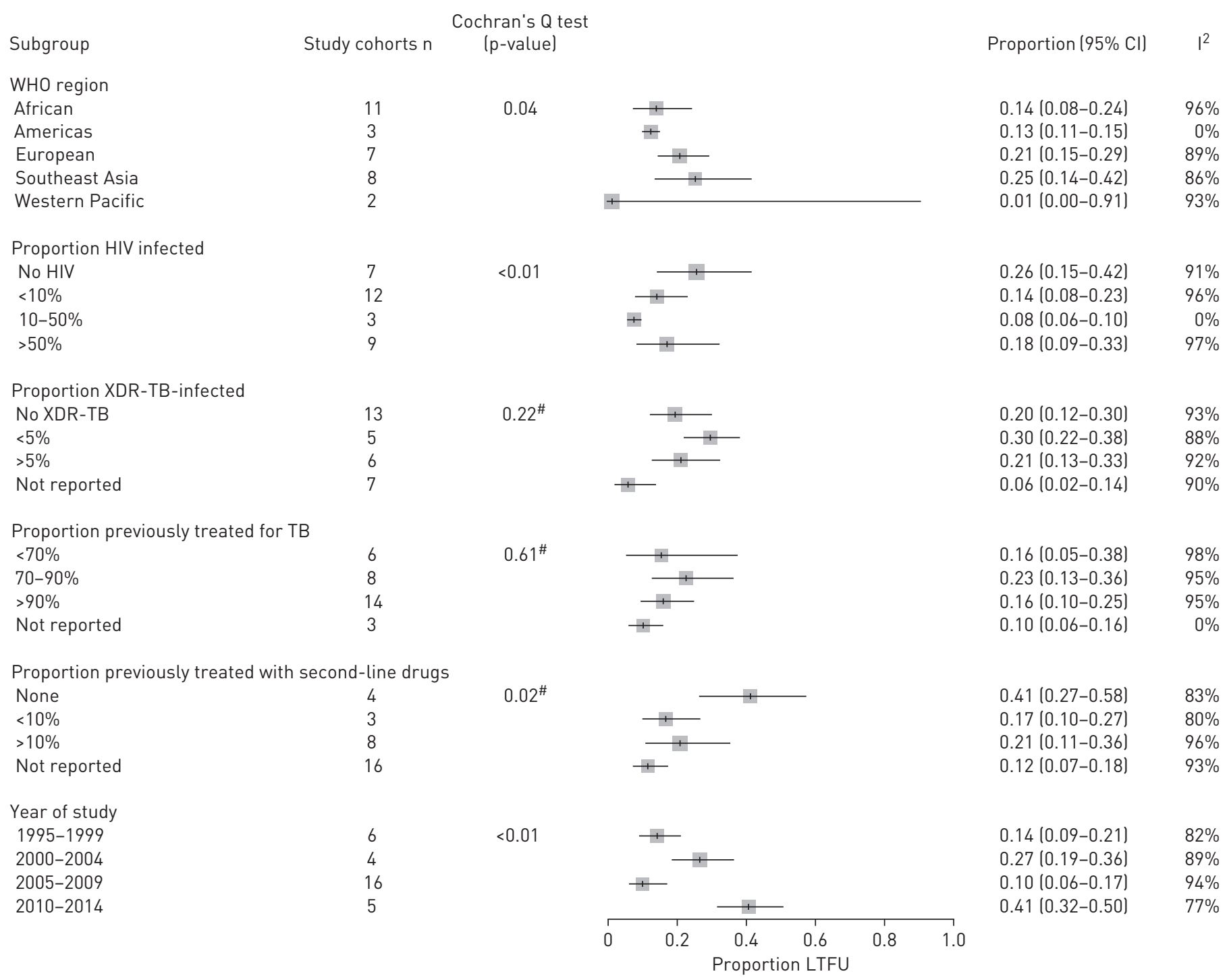

FIGURE 4 Forest plot of pooled proportions lost to follow-up (LTFU) stratified by study cohort characteristics. Patients who died, failed treatment, who transferred out or whose treatment outcome was not evaluated were excluded. WHO: World Health Organization; XDR: extensively drug-resistant; TB: tuberculosis. \#: study cohorts that did not report a given characteristic were excluded from Cochran's Q-test for subgroup differences. 


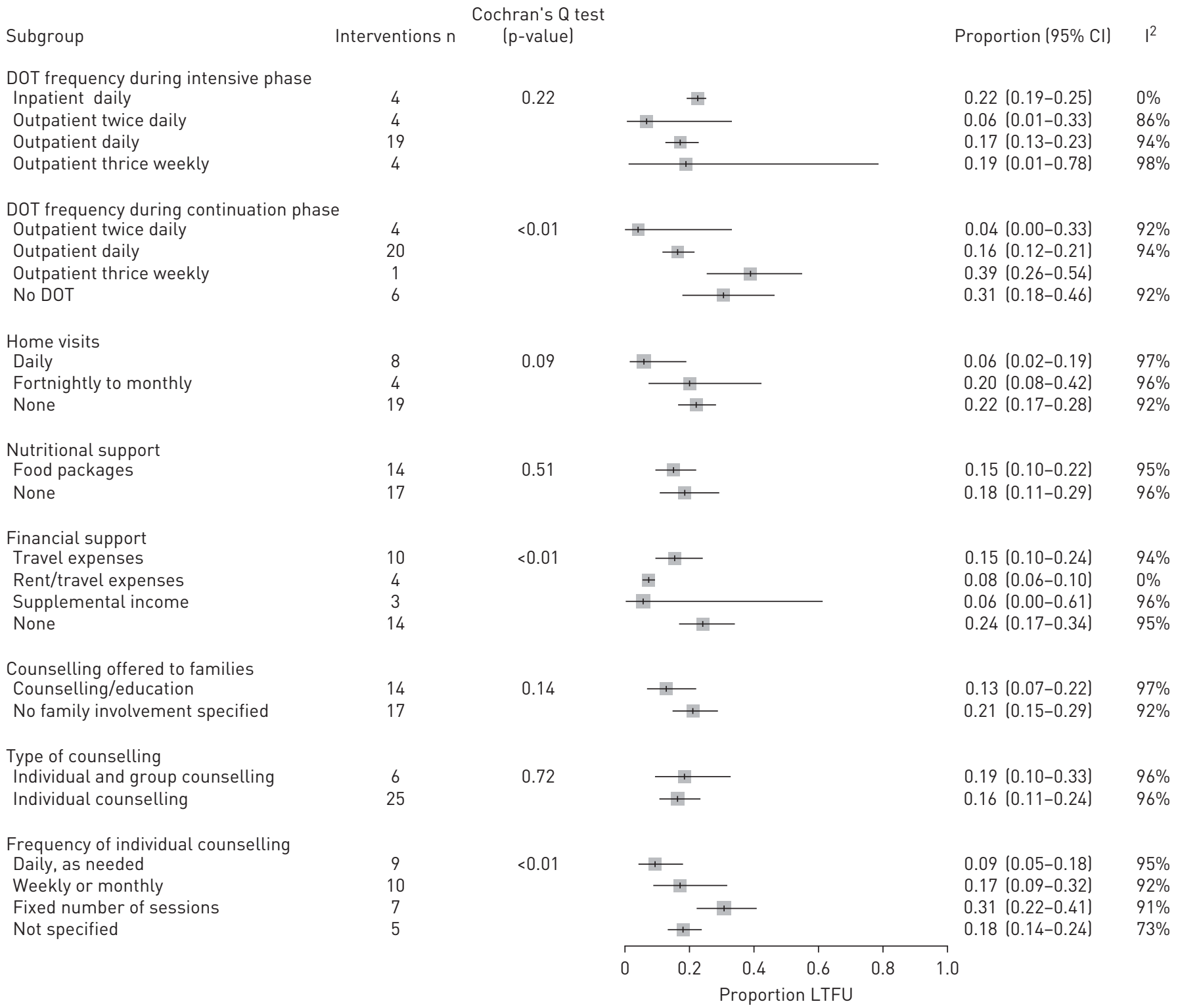

FIGURE 5 Forest plot of pooled proportions lost to follow-up (LTFU) stratified by frequency of directly observed therapy (DOT) during the intensive and continuation phase, and by type of adherence support provided during treatment. Patients who died, failed treatment, who transferred out or whose treatment outcome was not evaluated were excluded.

allocated to each patient was increased. These findings are consistent with those reported in a recent Cochrane systematic review [38], which found that daily DOT did not improve TB cure rates, compared to self-administered therapy, when the frequency of contact with providers increased from monthly to every 2 weeks or more.

In addition, this review provides evidence to support the effectiveness of financial compensation for rent or travel expenses, as well as lost wages, but not of group counselling, involvement of family in counselling sessions or nutritional support, on improving retention in care. The lack of effectiveness of those strategies could be due to residual confounding. For example, Cox et al. [14] found no effect of a community-based pilot intervention (which provided routine counselling and access to a peer support group), and suggested that this may be due to the higher numbers of patients who initiated treatment under intervention, who otherwise would not have received treatment. Furthermore, very few studies reported on implementation issues and fidelity of intervention delivery $[16,17,23,28,29,34,35]$. Thus, the reported intervention effectiveness may reflect issues with delivery, such as low engagement of patients and families in support groups [39]; lack of buy-in from health workers [16, 17, 32, 40]; or providers selectively providing adherence incentives, such as food packages, to patients deemed most worthy [41]. Future research should explore these issues through process evaluations [42]. 
Subgroup

Frequency of individual counselling

Daily, as needed

Weekly or monthly

Fixed number of sessions

Not specified

Home visits

Daily

Fortnightly to monthly

None

Nutritional support

Food packages

None

Financial support

Travel expenses

Rent/travel expenses

Supplemental income

None

Counselling offered to families

Counselling/education

No family involvement specified

Type of counselling

Individual and group counselling

Individual counselling

\section{Study cohorts Cochran's Q test}

(p-value)

Proportion $(95 \% \mathrm{CI})$

$1^{2}$

$0.09(0.05-0.18) \quad 95 \%$

$0.10(0.06-0.15) \quad 62 \%$

$0.30(0.20-0.42) \quad 94 \%$

$0.16(0.11-0.23) \quad 67 \%$

$0.06(0.02-0.19) \quad 97 \%$

$0.07(0.05-0.10) \quad 0 \%$

$0.20(0.15-0.26) \quad 91 \%$

$0.13(0.09-0.19) \quad 94 \%$

$0.13(0.06-0.27) \quad 97 \%$

$0.14(0.07-0.24) \quad 94 \%$

$0.08(0.06-0.10) \quad 0 \%$

$0.01(0.00-0.68) \quad 88 \%$

$0.21(0.14-0.30) \quad 94 \%$

$0.10(0.05-0.19) \quad 97 \%$

$0.18(0.13-0.25) \quad 91 \%$

$0.19(0.10-0.33) \quad 96 \%$

$0.12(0.08-0.19) \quad 95 \%$

$6 \quad 0.29$

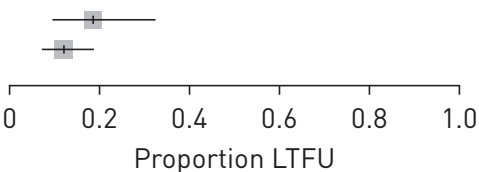

\section{0}

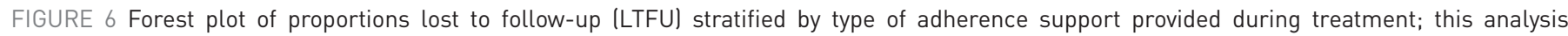

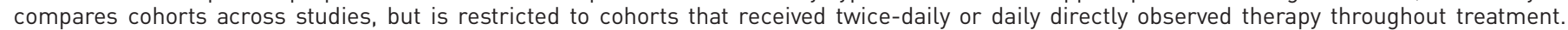
Patients who died, failed treatment, who transferred out or whose treatment outcome was not evaluated were excluded.

One major limitation of this review was the sparse descriptions of interventions provided by many studies. We made extensive efforts to contact authors for details, although we were not always successful. Among studies for which we were able to acquire sufficient details, we observed a wide variation in the educational and psychosocial support provided. Although nearly all studies provided individual counselling to patients, the specifics of the counselling were not often described sufficiently to gauge whether it encompassed social and emotional support and/or treatment education. Thus, we considered individual counselling to broadly include any one-on-one time spent between patients and health workers to address psychological, social or treatment-related issues, which could include both psychosocial and educational support, and was identified by the authors as beyond the current standard of care at the study sites. The observed benefit from these forms of support could therefore have resulted from added interactions between patients and their providers, beyond DOT and routine medical check-ups. However, it is difficult to assess the degree or depth of these interactions. Additionally, the benefit could be underestimated due to the lack of training provided in some studies compared to others.

Despite these limitations, the review provides a timely update on strategies to improve MDR-TB treatment retention in care, including results of two recent cluster randomised trials $[13,18]$. Unlike the earlier review [3], which included all studies reporting treatment outcomes for RR-/MDR-TB patients, we restricted our analysis to only those studies that explicitly provided patients with some form of psychosocial, educational or material support, allowing a more nuanced analysis comparing the effectiveness of different types of support. Notably, no interventions utilised e-health tools to promote adherence to RR-/MDR-TB treatment.

Our review provides the motivation for further examination of adherence interventions in RR-/MDR-TB, preferably through randomised controlled trials that compare the effectiveness of DOT to self-administered therapy, coupled with increased psychosocial and economic support throughout the treatment course. As evidenced by some recent cohort studies, and supported by expert commentaries [43, 44], a shift to self-administered therapy has the potential to relieve health worker burden so that their time and resources may be utilised to build health literacy, empower patients and deliver higher quality, patient-centred care. 
Acknowledgements: We would like to thank McGill University librarian Genevieve Gore (Montreal, Canada), for refining our search strategy.

Conflict of interest: None declared.

Support statement: This work was supported by the Canadian Institutes of Health Research (FRN143999). S. Law was supported by a Vanier Canada Graduate Scholarship. Funding information for this article has been deposited with the Crossref Funder Registry.

\section{References}

$1 \quad$ World Health Organization (WHO). Global Tuberculosis Report 2017. Geneva, WHO, 2017.

2 Munro SA, Lewin SA, Smith HJ, et al. Patient adherence to tuberculosis treatment: a systematic review of qualitative research. PLoS Med 2007; 4: e238.

3 Toczek A, Cox $\mathrm{H}$, du Cros $\mathrm{P}$, et al. Strategies for reducing treatment default in drug-resistant tuberculosis: systematic review and meta-analysis. Int J Tuberc Lung Dis 2013; 17: 299-307.

4 Moher D, Liberati A, Tetzlaff J, et al. Preferred reporting items for systematic reviews and meta-analyses: the PRISMA Statement. PLoS Med 2009; 6: e1000097.

5 Stroup DF, Berlin JA, Morton SC, et al. Meta-analysis of observational studies in epidemiology: a proposal for reporting. JAMA 2000; 283: 2008-2012.

6 World Health Organization (WHO). Guidelines for Establishing DOTS-Plus Projects for the Management of Multidrug-Resistant Tuberculosis (MDR-TB). Geneva, WHO, 2000.

7 Ho J, Byrne AL, Linh NN, et al. Decentralized care for multidrug-resistant tuberculosis: a systematic review and meta-analysis. Bull World Health Organ 2017; 95: 584-593.

8 Weiss $\mathrm{P}$, Chen $\mathrm{W}$, Cook VJ, et al. Treatment outcomes from community-based drug resistant tuberculosis treatment programs: a systematic review and meta-analysis. BMC Infect Dis 2014; 14: 333.

9 Yin J, Yuan J, Hu Y, et al. Association between directly observed therapy and treatment outcomes in multidrug-resistant tuberculosis: a systematic review and meta-analysis. PLoS One 2016; 11: e0150511.

10 Sterne J, Hernán MA, Reeves BC, et al. ROBINS-I: a tool for assessing risk of bias in non-randomised studies of interventions. BMJ 2016; 355: i4919.

11 Higgins JPT, Green S, Eds. Cochrane Handbook for Systematic Reviews of Interventions Version 5.1.0. London, The Cochrane Collaboration, 2011.

12 Hamza TH, van Houwelingen HC, Stijnen T. The binomial distribution of meta-analysis was preferred to model within-study variability. J Clin Epidemiol 2008; 61: 41-51

13 Baral SC, Aryal Y, Bhattrai R, et al. The importance of providing counselling and financial support to patients receiving treatment for multi-drug resistant TB: mixed method qualitative and pilot intervention studies. BMC Public Health 2014; 14: 46.

14 Cox H, Hughes J, Daniels J, et al. Community-based treatment of drug-resistant tuberculosis in Khayelitsha, South Africa. Int J Tuberc Lung Dis 2014; 18: 441-448.

15 Huerga $\mathrm{H}$, Bastard $\mathrm{M}$, Kamene $\mathrm{M}$, et al. Outcomes from the first multidrug-resistant tuberculosis programme in Kenya. Int J Tuberc Lung Dis 2017; 21: 314-319.

16 Loveday $\mathrm{M}$, Wallengren $\mathrm{K}$, Brust $\mathrm{J}$, et al. Community-based care vs. centralised hospitalisation for MDR-TB patients, KwaZulu-Natal, South Africa. Int J Tuberc Lung Dis 2015; 19: 163-171.

17 Mohr E, Daniels J, Beko B, et al. DOT or SAT for rifampicin-resistant tuberculosis? A non-randomized comparison in a high HIV-prevalence setting. PLoS One 2017; 12: e0178054.

18 Taneja N, Chellaiyan VG, Daral S, et al. Home based care as an approach to improve the efficiency of treatment for MDR tuberculosis: a quasi-experimental pilot study. J Clin Diagn Res 2017; 11: LC05-LC08.

19 Alene KA, Yi HZ, Viney K, et al. Treatment outcomes of patients with multidrug-resistant and extensively drug resistant tuberculosis in Hunan Province, China. BMC Infect Dis 2017; 17: 573.

20 Bastard M, Sanchez-Padilla E, Hewison C, et al. Effects of treatment interruption patterns on treatment success among patients with multidrug-resistant tuberculosis in Armenia and Abkhazia. J Infect Dis 2015; 211: 1607-1615.

21 Cox HS, Kalon S, Allamuratova S, et al. Multidrug-resistant tuberculosis treatment outcomes in Karakalpakstan, Uzbekistan: treatment complexity and XDR-TB among treatment failures. PLoS One 2007; 2: E1126.

22 Escudero E, Peña JM, Alvarez-Sala R, et al. Multidrug-resistant tuberculosis without HIV infection: success with individualised therapy. Int J Tuberc Lung Dis 2006; 10: 409-414.

23 Gelmanova IY, Taran DV, Mishustin SP, et al. 'Sputnik': a programmatic approach to improve tuberculosis treatment adherence and outcome among defaulters. Int J Tuberc Lung Dis 2011; 15: 1373-1379.

24 Isaakidis P, Cox HS, Varghese B, et al. Ambulatory multi-drug resistant tuberculosis treatment outcomes in a cohort of HIV-infected patients in a slum setting in Mumbai, India. PLoS One 2011; 6: e28066.

25 Joseph P, Desai VB, Mohan NS, et al. Outcome of standardized treatment for patients with MDR-TB from Tamil Nadu, India. Indian J Med Res 2011; 133: 529-534.

26 Keshavjee S, Gelmanova IY, Farmer PE, et al. Treatment of extensively drug-resistant tuberculosis in Tomsk, Russia: a retrospective cohort study. Lancet 2008; 372: 1403-1409.

27 Kliiman K, Altraja A. Predictors of poor treatment outcome in multi- and extensively drug-resistant pulmonary TB. Eur Respir J 2009; 33: 1085-1094.

28 Meressa D, Hurtado RM, Andrews JR, et al. Achieving high treatment success for multidrug-resistant TB in Africa: initiation and scale-up of MDR TB care in Ethiopia - an observational cohort study. Thorax 2015; 70: 1181-1188.

29 Mitnick C, Bayona J, Palacios E, et al. Community-based therapy for multidrug-resistant tuberculosis in Lima, Peru. New Engl J Med 2003; 348: 119-128.

30 Mitnick CD, Shin SS, Seung KJ, et al. Comprehensive treatment of extensively drug-resistant tuberculosis. $N$ Engl J Med 2008; 359: 563-574.

31 Mohr E, Cox V, Wilkinson L, et al. Programmatic treatment outcomes in HIV-infected and uninfected drug-resistant TB patients in Khayelitsha, South Africa. Trans R Soc Trop Med Hyg 2015; 109: 425-432. 
32 Satti H, McLaughlin MM, Hedt-Gauthier B, et al. Outcomes of multidrug-resistant tuberculosis treatment with early initiation of antiretroviral therapy for HIV co-infected patients in Lesotho. PLoS One 2012; 7: e46943.

33 Shin SS, Pasechnikov AD, Gelmanova IY, et al. Treatment outcomes in an integrated civilian and prison MDR-TB treatment program in Russia. Int J Tuberc Lung Dis 2006; 10: 402-408.

34 Suárez PG, Floyd K, Portocarrero J, et al. Feasibility and cost-effectiveness of standardised second-line drug treatment for chronic tuberculosis patients: a national cohort study in Peru. Lancet 2002; 359: 1980-1989.

35 Thomas A, Ramachandran R, Rehaman F, et al. Management of multi drug resistance tuberculosis in the field: Tuberculosis Research Centre experience. Indian J Tuberc 2007; 54: 117-124.

36 Vaghela JF, Kapoor SK, Kumar A, et al. Home based care to multi-drug resistant tuberculosis patients: a pilot study. Indian J Tuberc 2015; 62: 91-96.

37 Yu MC, Chen HY, Chien SH, et al. An integrated MDR-TB management programme results in favourable outcomes in northern Taiwan. Eur Respir J 2015; 45: 272-275.

38 Karumbi J, Garner P. Directly observed therapy for treating tuberculosis. Cochrane Database Syst Rev 2015; 5: CD003343.

39 Acha J, Sweetland A, Guerra D, et al. Psychosocial support groups for patients with multidrug-resistant tuberculosis: five years of experience. Glob Public Health 2007; 2: 404-417.

40 Brust JC, Shah NS, Scott M, et al. Integrated, home-based treatment for MDR-TB and HIV in rural South Africa: an alternate model of care. Int J Tuberc Lung Dis 2012; 16: 998-1004.

41 Lutge E, Lewin S, Volmink J, et al. Economic support to improve tuberculosis treatment outcomes in South Africa: a pragmatic cluster-randomized controlled trial. Trials 2013; 14: 154.

42 Munro A, Bloor M. Process evaluation: the new miracle ingredient in public health research? Qual Res 2010; 10: 699-713.

43 Metcalfe JZ, O’Donnell MR, Bangsberg DR. Moving beyond directly observed therapy for tuberculosis. PLoS Med 2015; 12: e1001877.

44 Benbaba S, Isaakidis P, Das M, et al. Direct observation (DO) for drug-resistant tuberculosis: do we really DO? PLoS One 2015; 10: e0144936. 